\title{
Un programa de educación en valores. La formación moral en alumnos de educación secundaria obligatoria
}

\author{
Roberto Sanz Ponce ${ }^{1}$
}

José M. Mula Benavent ${ }^{2}$

\begin{abstract}
Resumen
La expresión "educar en valores" puede parecer, a primera vista, redundante, pues se piensa que no es posible educar de otra manera. No obstante, no basta con que el profesorado proclame los valores, sino que es necesaria la implementación de unos determinados estilos docentes que favorezcan las relaciones entre profesores y alumnos y de éstos con los contenidos curriculares.
\end{abstract}

El ámbito familiar no debería ser ajeno a esta realidad. Así pues, todo intento serio de elaborar un programa en valores debe tener como base fundamental una adecuada y sólida formación pedagógica del profesorado, con un buen dominio de técnicas y estrategias específicas para la educación en valores. Por ello, se plantea la necesidad de presentar, de manera personalizada -grupal e individualmente- un programa de educación en valores para alumnos de Educación Secundaria Obligatoria de la ciudad de Valencia, que pueda dar respuesta a las necesidades de los sujetos destinatarios de la misma.

Palabras clave: actitud y valores, aprendizaje de valores, educación en valores, plan de acción tutorial.

\footnotetext{
1 Doctor en Ciencias de la Educación. Profesor Agregado de la Universidad Católica de Valencia "San Vicente Mártir." Vicedecano de Pedagogía. Director de la Revista Edetania. Estudios y propuestas socioeducativas. Secretario del Departamento de Ciencias de la Educación. Secretario del Instituto Universitario de Teoría de la Educación de la Universidad Católica de Valencia. roberto.sanz@ucv.es
} 


\title{
Education in values program. Moral formation in secondary education students
}

\begin{abstract}
The expression "educating in values" may seem redundant at first sight, because we think it's impossible to educate in a different way. However, it's not enough that the teachers declare the values, it would be necessary to implement a certain teaching styles that would promote the relations between teachers and students, and between them and the curriculum content. The family enviroment should not be oblivious to this reality. Thus, any serious attempt to develop a program on values should have as a fundamental base an adequate and solid pedagogical formation of teachers, with a good knowledge of specific techniques and strategies for the education in human values. Therefore, there is a need to present in a personalized way - both for groups and individuals - a program of values education for pupils of Compulsory Secondary Education in the city of Valencia, which can respond to the needs of the recipients of the same.
\end{abstract}

Key words: attitude and values, teaching values, tutorial plan, values education.

\section{lntroducción}

La adolescencia siempre ha sido considerada como una etapa psicoevolutiva compleja, donde el adolescente se enfrenta a una búsqueda de su propia identidad personal -normalmente en contraposición a la de los padres,- y de referentes que le ayuden a superar esa cierta inestabilidad emocional que se produce en el difícil tránsito de la infancia a la juventud. Esta "etapa de crisis," en la que se pone en tela de juicio tanto los valores como las creencias y los principios, hasta entonces estables y seguros, en búsqueda de esa ansiada autonomía e independencia, otorgan a este periodo de edad una importancia capital para la educación en valores. 
Ya la UNESCO, en los años 90, dotaba a la Educación un carácter eminentemente axiológico. En su conocido informe La educación encierra un tesoro (1996) la Comisión presidida por Jacques Delors, describía los cuatro pilares fundamentales para la educación en el siglo XXI. Entre estos pilares destacan el aprender a convivir y el aprender a ser, pilares que, lógicamente, se cimientan sobre una sólida educación en valores. El citado informe dirá: "Se trata de aprender a vivir juntos conociendo mejor a los demás, su historia, sus tradiciones y su espiritualidad y, a partir de ahí, crear un espíritu nuevo que impulse la realización de proyectos comunes o a la solución inteligente y pacífica de los inevitables conflictos..." (Delors, 1996: 18).

Por ello, creemos que la escuela debe convertirse no solo en una agencia transmisora de conocimientos, sino en una agencia portadora-vivencial- de ciertos valores y principios morales que deben ser aprendidos y aprehendidos por los alumnos. De esta manera, el docente debe asumir el rol de "educador," convirtiéndose en un referente de su episteme, asignatura o área de conocimiento, además de en un modelo de conducta y de actuación moral. No se olvide que, muchas veces, nuestros alumnos aprenden más de nuestros actos y comportamientos que de nuestras palabras. El propio Dewey, en Democracia y educación, mantenía que: "El ejemplo es notoriamente más poderoso que el precepto. Las buenas maneras proceden, como se dice, de la buena crianza, o mejor son buena crianza; y ésta se adquiere por la acción habitual, en respuesta a estímulos habituales no por una información transmitida" (1995:27). Además, la escuela actual se enfrenta a nuevos problemas/desafíos.

La familia, en algunas ocasiones, ha comenzado peligrosamente a hacer dejación de sus funciones y obligaciones educativas para con sus hijos, abandonando su papel como primera institución formativa. El aumento en el número de familias en las que ambos cónyuges trabajan fuera del hogar y la cada vez más temprana incorporación de los niños al sistema educativo (Educación Infantil de 0-3 y 3-6 años) ha restado autoridad educativa a las familias; así mismo, la influencia de unos medios de comunicación -televisión, internet, videojuegos, ...- más preocupados por los índices de audiencias o por el número de ventas de sus productos, que por el nivel moral de los contenidos emitidos, también, están dificultando la tarea educativa de la escuela.

Ante esta realidad, cada vez más palpable y extendida, entendemos la necesidad de diseñar una intervención decidida, planificada y sistematizada, con los jóvenes en edad escolar, en temas relacionados con la educación moral. La creación de un Programa de educación en valores que intente paliar las deficiencias y necesidades de una sociedad cada día más preocupada por el "tener" frente al "ser" -cuánto tienes cuánto vales-; con jóvenes atrapados en 
un consumismo desmesurado -adicciones de todo tipo- con un clima social de egoísmo personal, con una clara falta de identidad de sus gentes, con carencias importantes en temas como la libertad y la responsabilidad, se plantea como urgencia educativa para la escuela del siglo XXI.

\section{Algunas notas para la educación en valores}

¿Para qué una educación en valores? El profesor Escámez, ya en 1986, se planteaba que la educación en actitudes y valores era una exigencia para el hombre de hoy - ayer, argumento que no ha perdido validez con el paso de los años. En ese primer capítulo, al que hacíamos mención, recoge cómo la legislación y las instituciones -nacionales e internacionales- confieren a la educación en valores una importancia capital, aunque también matiza cuáles son las dificultades y obstáculos ante los que se enfrenta. Ayer igual que hoy -apunta Escámez- el hombre "Está siendo bombardeado por un número incesantemente creciente de estímulos situacionales a los que, tanto desde un punto de vista personal como socio-laboral, se encuentra obligado a responder; estos estímulos situacionales son contextos desde donde aprende, "red de aprendizaje" en expresión de Coombs (1985), a los que se debe responder, desde una óptica educativa, de un modo adecuado" (Escámez \& Ortega, 1986: 14). Han pasado los años, han cambiado sustancialmente las circunstancias, incluso se ha atravesado, en España, por tres nuevas leyes educativas (LOGSE, LOCE y LOE) y el discurso continua vigente y actual, continua planteándose como un reto o desafío al que debe dar respuesta la escuela.

Pero, tal vez, antes de abordar el tema de la educación en valores, se debería plantear una serie de interrogantes que nos ayudasen a situar la cuestión de estudio. En primer lugar, definir qué se entiende por Educación en su sentido más amplio y cuál es el papel de la escuela en el proceso de aprendizajeenseñanza. En segundo lugar, analizar el concepto de Valor en términos generales, para plantear, en tercer lugar, qué es la educación en valores y para qué sirve en una sociedad como la actual.

Para nosotros, la educación es entendida como un proceso a través del cual el ser humano se va "autoeducando," es decir, por el que va adquiriendo las competencias, habilidades, destrezas y valores necesarios para desenvolverse en su vida. En ese proceso, la escuela juega un papel fundamental, junto con la familia y la sociedad en general, como generadora de "situaciones de aprendizaje". Es cierto que las finalidades del sistema educativo han ido variando en el transcurso de los años (Sanz, 2011), más si cabe en la etapa educativa de Educación Secundaria que ha pasado, en los últimos años, de ser un tramo voluntario, propedéutico y selectivo a convertirse en un tramo obligatorio, general y básico, con todas las consecuencias que eso conlleva. 
En este nuevo escenario, toma sentido la educación en valores, aunque, tal vez nunca dejó de tenerlo. ${ }^{3}$ La educación siempre ha sido en valores, ya que sin valores no hay educación (Reboul, 2009). Los profesores Martín y Puig Rovira, afirman que "La intención última de la educación en valores es ayudar a los chicos y chicas a aprender a vivir. Es la primera tarea de los seres humanos porque, a pesar de estar preparados para vivir, necesitamos adoptar una forma de vida que sea posible sostener y que realmente queramos para nosotros y para todos los que nos rodean" (2007: 16). Todavía es más urgente, tal y como mantiene Vinuesa, en una sociedad en constante cambio, en plena encrucijada, en la que "Debemos salvar de la quema los valores que siguen siendo válidos para una sociedad distinta en un futuro no lejano" (2002: 61).

Los valores sirven como patrones para guiar la vida de las personas, ya que son la razón y el fundamento por el que una persona elige hacer o dejar de hacer una determinada acción o reacciona de una manera $u$ otra ante un acontecimiento personal o social. El sistema de valores de una persona es el punto de referencia para evaluar las situaciones, el modo de resolver conflictos y la manera de tomar decisiones. Éstos se van conformando como resultado de la interacción de la persona con su ambiente, lo que tiene un interés fundamental para la educación, ya que va a permitir que el alumno vaya realizando el aprendizaje de los mismos, y evolucionando paulatinamente hacia su apropiación. La escuela trata de dar respuesta a estas demandas, pero generalmente con recursos específicos escasos (Mula, 2007). ${ }^{4}$

Además, la educación en valores se enfrenta ante una serie de miedos o problemas: el conformismo social y el adoctrinamiento. En palabras del profesor Escámez "Los profesores y educadores sienten ciertos temores: para no ir más allá de lo que pueda corresponderles, para no invadir un ámbito al que quizás no tengan derecho, para no asumir la conducción de otra persona, anulando su derecho a regirse por ella misma" (1998: 54). En definitiva, tratar de huir del fantasma del adoctrinamiento.

A estos problemas se suma la resistencia por parte de algunos profesores normalmente por falta de formación- a enfrentarse a una educación en valores, así como, un cierto menosprecio de algunos padres hacia este tipo de enseñanzas, a las que consideran como una "pérdida de tiempo." Pero a pesar de todos estos inconvenientes, miedos, temores y resistencias surge con fuerza la necesidad de corresponsabilidad del sistema escolar y de los propios docentes en la construcción de una sociedad más humana y más justa, sociedad

3 La profesora Adela Cortina explica que la educación en valores no es un tema de moda, sino que se trata de una cuestión de actualidad, que siempre y en toda sociedad está presente de una u otra forma, ya que "Forma parte de nuestro ser más profundo, de nuestra más entrañable realidad, además de ser importante de suyo, ha salido a la luz por uno u otro motivo y las gentes han dado en hablar de él. No radica tanto su importancia entonces en que ande de boca en boca, sino en que es un ingrediente indispensable de la vida humana, inseparable de nuestro ser persona" (1996: 2). 
que únicamente podrá construirse si se cimienta sobre un clima "moral en el aula"-democrático, participativo, libre-, que favorezca un verdadero aprendizaje de los valores.

\section{¿Qué debemos enseñar?}

Sin entrar en consideraciones más específicas de cuáles deben ser los valores que deben enseñarse y aprenderse en el aula, se parte aquí de una idea fundamental. La educación en valores que debe proporcionar la escuela es aquella que humaniza al ser humano y lo prepara para con-vivir en una sociedad democrática, plural y libre. "Educar en valores es educar moralmente, porque son los valores los que enseñan al individuo a comportarse como hombre, establecer una jerarquía entre las cosas, llegar a la convicción de que algo importa o no importa, vale o no vale, es un valor o un contravalor" (Carreras y otros, 1996: 22). Se trata de educar en valores comunes, que deban y merezcan ser aprendidos por todos, a pesar de las diferencias que nos distinguen.

En esa misma línea, Reboul, en su obra de Filosofía de la Educación (2009), mantiene que se debe centrar la educación en valores en dos parámetros: "aquello que une" y "aquello que libera." Lo que une hace referencia a aquello que es duradero en el ser humano y en la sociedad, aquello que perdura y no está sujeto a modas. Por su parte, lo que libera se refiere a aquella educación que nos hace libres, conocedores de las diferentes opciones existentes y capaces de elegir alguna de ellas con libertad y responsabilidad. En definitiva, se debe enseñar aquellos valores que provoquen la formación/creación de una persona y de una sociedad más justa, solidaria, libre, responsable y autónoma.

\section{¿A quién corresponde educar en valores? El papel del docente}

La educación en valores es tarea de todos los que actúan, de un modo u otro, sobre los educandos. Pero, aunque exista una corresponsabilidad de todos los estamentos, parece que, de hecho, los espacios más adecuados para llevarla a término sean la familia y la escuela, sin olvidar el espacio creciente que ocupa la educación no formal. Si se considera los valores como contenidos de aprendizaje, parece lógico pensar que la escuela reúne las condiciones para ser el espacio ideal para su enseñanza. Si se les considera como patrones referentes de conducta, serán las experiencias vividas por el sujeto en la familia y las relaciones que se presentan el cauce a través del cual se produce la transmisión de valores y contravalores.

Debido a este carácter dual y fronterizo, cabe matizar ciertas consideraciones (Mula, 2007): 
- La escuela no puede omitir su responsabilidad de educar a los alumnos en los valores que haya elegido como propios y distintivos y que, por tanto, figuran en su Proyecto Educativo de Centro.

- En el entorno familiar en el que vive el alumno debe comenzarse la educación en valores. Como consecuencia de esta afirmación, los profesores deben trabajar conjuntamente con las familias.

- El profesor es una persona relevante para sus alumnos. Su conducta, el afecto, la aceptación y la comprensión con las que se desarrollen sus relaciones con los alumnos, van a ser factores determinantes en el aprendizaje de los valores por parte de éstos.

- El alumno tiende a identificar la experiencia de un valor con el modelo más cercano que se lo propone. Por ello, es fundamental que las personas que actúan como modelo, padres, profesores y personas significativas de su entorno, le ofrezcan modos de comportamiento positivos que puedan servir de referente.

De esta manera, "La tarea de educar y, con ello, la de educar en valores, no queda circunscrita - todos lo sabemos- al ámbito escolar. Familia y sociedad son espacios sociales fuertemente comprometidos en esta responsabilidad. Ahora bien, hay deberes que afectan directamente a la escuela si a ésta se le asigna una múltiple razón de ser: orientación a los alumnos en la búsqueda e interpretación de la realidad, ayuda al desarrollo de su personalidad a través de un proceso dinámico que pone en juego sus capacidades y actitudes,..." (Bartolomé et al., 1979: 206). Aunque como se ha dicho familia y sociedad no siempre parecen asumir sus responsabilidades.

Por tanto, queda a la escuela la difícil tarea de asumir la responsabilidad de la educación en valores. En ese escenario, surge la figura del docente como una pieza fundamental. Aunque, esta obviedad, que para las personas que tienen cierta relación con el mundo escolar es totalmente lógica, debe ser matizada. Es cierto, como se ha dicho, que la figura del docente es muy importante en el proceso de formación de la persona, aunque esa importancia/ influencia puede ser positiva o negativa en función de las cualidades, actitudes y comportamientos que los docentes muestren en el aula y fuera de ella. Son numerosos los estudios que analizan la influencia de los profesores en la adquisición de los valores morales por parte de los alumnos. Éstos se enfrentan ante el gran desafío de Ser (modelo de referencia) y de Dejar Ser (favorecer la maduración de los alumnos), convirtiendo la educación en un proceso de guía y orientación y no de imposición e inculcación. Por ello, y sin desviarnos del tema que se está trabajando, se enuncian una serie de características docentes que benefician el aprendizaje de los valores. He aquí algunas de ellas: 
Características personales: En el aula se suceden un gran número de interrelaciones personales que influyen y determinan el aprendizaje en y de valores de los alumnos. El autocontrol, entendido como la capacidad de dominar ciertas situaciones -emociones, sentimientos e incluso conductas;poseer una equilibrada autoestima, tener empatía, y ser asertivo, son algunas de estas características personales que ayudan a la adquisición de valores de forma vicaria.

Características pedagógicas: La creación de un clima de confianza y de respeto, que fomente la comunicación interpersonal y la participación de nuestros alumnos en clase, también estimula el aprendizaje de ciertos valores, fundamentalmente el del respeto y el de la libertad a expresar las opiniones. En definitiva, vivir lo que se enseña, creerse el papel del maestro como aquel que modela, guía y orienta a su aprendiz. Divertirse enseñando y mostrar que el esfuerzo y la dedicación dan su fruto, forja caracteres muy determinados entre los alumnos.

\section{Un programa para alumnos de Educación Secundaria Obligatoria}

Realizar un Programa de educación en valores enfocado a la escuela y, concretamente a la Enseñanza Secundaria, ${ }^{5}$ tiene su fundamento en lo que vienen a afirmar los profesores Martín y Puig Rovira: "Enseñar a vivir no es transmitir saberes, sino un "saber hacer." Es así porque lo que se requiere es el dominio de un conjunto de habilidades, capacidades o virtudes. Y todo esto no se aprende con discursos y de memoria, sino mediante la observación, la práctica, el ejercicio y el refuerzo que otorgue a las personas apreciadas. Enseñar a vivir apunta a la formación de ciertas disposiciones y capacidades: un "saber hacer." Y la adquisición de este dominio hace imprescindible el ejercicio y el entrenamiento: la participación activa en prácticas de valor propias de una comunidad. Enseñar a vivir no sólo transmite un "saber hacer," sino también estimación y pasión por este "saber hacer” (2007: 19).

De esta manera, se considera que la necesidad de formar en valores viene justificada por tres imperativos fundamentales: a) El desarrollo de una educación integral del alumno que conlleva, entre otros, la adquisición del conocimiento de valores concretos, de unos hábitos para la convivencia y el desarrollo de la competencia de autonomía personal; b) Dar respuesta al vacío de valores que se constata actualmente en la sociedad, dando sentido a la propia existencia y posibilitando la convivencia y la adopción de modelos

5 La Educación Secundaria Obligatoria (ESO), en España, tiene carácter obligatorio, básico y gratuito; está dividido en cuatro cursos académicos: $1^{\circ}, 2^{\circ}, 3^{\circ}$ y $4^{\circ}$ y comprende las edades de 12 a 16 años. 
que orienten la actuación individual y colectiva; y c) Educar para el desarrollo de una ciudadanía responsable que vehiculase la participación activa en los distintos ámbitos sociales, el actuar de acuerdo con una escala de valores de forma ética, razonada y solidaria, que permita al individuo la elaboración dinámica de su proyecto de vida. Con esta idea y bajo este propósito planteamos este Programa de educación en valores para alumnos de ESO.

\section{Objetivos}

El Programa se compone de 12 ejes temáticos, que pretenden ser los puntos de referencia para la reflexión sobre el Saber Ser y el Saber Estar, es decir, para el desarrollo moral del alumno. Estos temas se trabajarán en la clase de tutoría, siendo el tutor el responsable de dirigir las actividades propuestas en las sesiones. Está estructurado en 4 fases, aunque su aplicación no debe ser necesariamente secuencial, ni identificarse con cursos académicos concretos. Las necesidades de los alumnos marcarán los itinerarios de implementación.
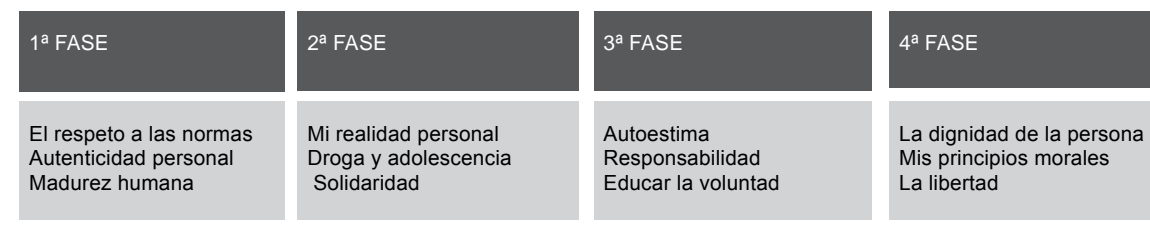

Los objetivos generales del Programa son:

- Desarrollar una visión ajustada de sí mismo y del otro, para desde ella organizar, tanto el saber ser, como el saber estar.

- Clarificar conceptos y términos relacionados con la educación en valores, dotándola de contenido y recursos, para poder actuar de forma coherente a las normas asumidas como propias.

- Analizar factores que influyen en la formación de las normas, para poder conformar las propias, y tomar decisiones de acuerdo con las mismas.

- Esforzarse en aplicar las normas configuradas como propias, para actuar en diferentes situaciones de forma moralmente buena.

-Responsabilizarse de las propias actuaciones, entendidas como manifestación del compromiso con el otro, para mejorar la sociedad.

\section{Competencias}

El programa desarrollado pretende contribuir a la adquisición por parte de los alumnos de Educación Secundaria Obligatoria de las siguientes competencias. 
Toma en consideración las necesidades y los intereses de los demás, así como los factores ligados a su contexto socioeconómico y cultural, para tomar decisiones acordes con las situaciones o exigencias que las mismas demandan.

○emuestra un espíritu crítico hacia sus propias convicciones, ideas, percepciones, juicios, prejuicios o preferencias culturales, para adoptar estrategias que le permitan actuar eficientemente en las situaciones de relación con los otros.

O Reflexiona sobre su práctica y reinvierte los resultados de la misma en la acción, para llevar a cabo proyectos coherentes de mejora personal y ciudadana.

Justifica sus decisiones, y la pertinencia de las mismas, en el ámbito personal, ciudadano y profesional, para asumir la responsabilidad de sus actuaciones como individuo y como miembro de la sociedad.

Es consciente de los principios éticos e implicaciones sociales de sus actuaciones, para afrontar situaciones problemáticas de forma coherente con sus principios morales.

Desarrollo del Programa de educación en valores: "Actuar de acuerdo con las propias convicciones."

Como se ha ido afirmando a lo largo del trabajo, un programa de educación en valores precisa de unas metodologías docentes concretas y específicas que favorezcan una adquisición real y vivencial en los alumnos. No hay que olvidar que el éxito de un Programa de Educación en Valores depende en gran parte, además del papel de la Escuela, de la colaboración en sus distintos niveles de implicación y de responsabilidad, de los diferentes agentes que inciden en los alumnos, como son: la familia y la sociedad, en tanto la influencia que ejercen el grupo de iguales y los medios de comunicación. Sin su ayuda y apoyo todo será más complejo.

El programa "Actuar de acuerdo a las propias convicciones" está pensado para alumnos de Secundaria. En su diseño se ha tenido en cuenta la importancia de intervenir, en su aplicación, no solo el tutor del grupo, sino también profesores de distintas asignaturas con actividades-tareas relacionadas con sus contenidos específicos. Para ello, se mantienen reuniones con los profesores del curso, intervengan o no directamente en las actividades de desarrollo previstas, para explicar la colaboración que se espera de ellos y, en el caso de aquellos docentes que participan desde sus respectivas asignaturas, además, dar las pautas pertinentes que van a orientar las actividades por realizar y su participación en las mismas. 
Así mismo, el programa prevé la realización de sesiones de trabajo con los padres de los alumnos, de forma que sean conocedores del desarrollo del programa y dotarles de recursos para poder reforzar y colaborar, desde el ámbito familiar, con los objetivos y actividades que sus hijos realizan en el centro. lo referente a las sesiones, se contemplan tanto actividades individuales como en equipo y en grupo, así como actividades de reflexión, de información y de autorregulación. En el desarrollo de las mismas se proponen distintas dinámicas y metodologías.

Como ejemplificación del desarrollo de las sesiones del programa se presentan las fichas correspondientes a las sesiones de trabajo de tres de ellas: "El respeto a las normas," "Autenticidad personal" y "Madurez humana." Todo el programa se encuentra vinculado al Plan de Acción Tutorial al atender tanto a los alumnos, como al profesorado y a las familias.

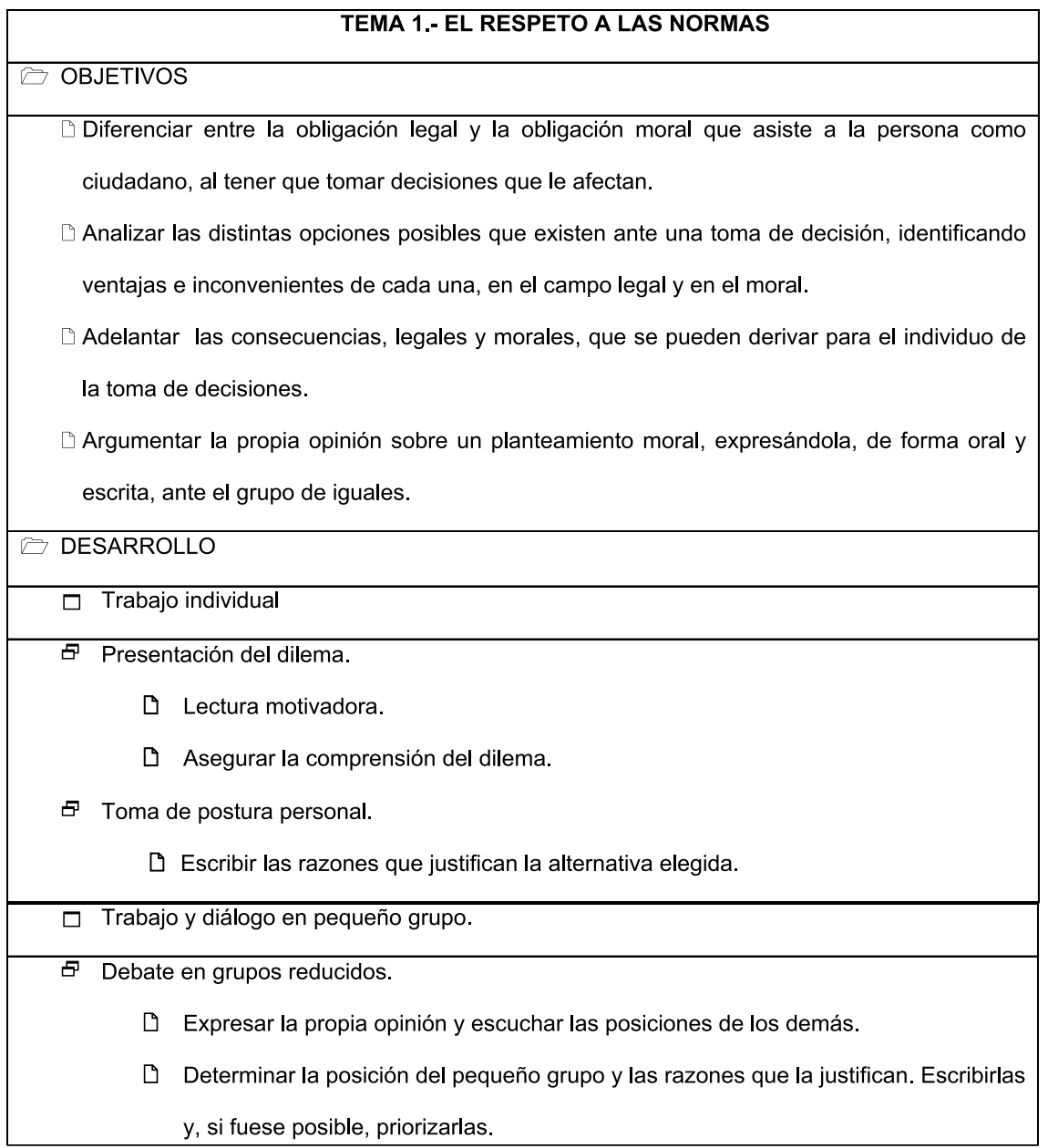




\section{Roberto Sanz Ponce y José M. Mula Benavent}

Un programa de educación en valores.

La formación moral en alumnos de educación secundaria obligatoria. Artículo de reflexión.

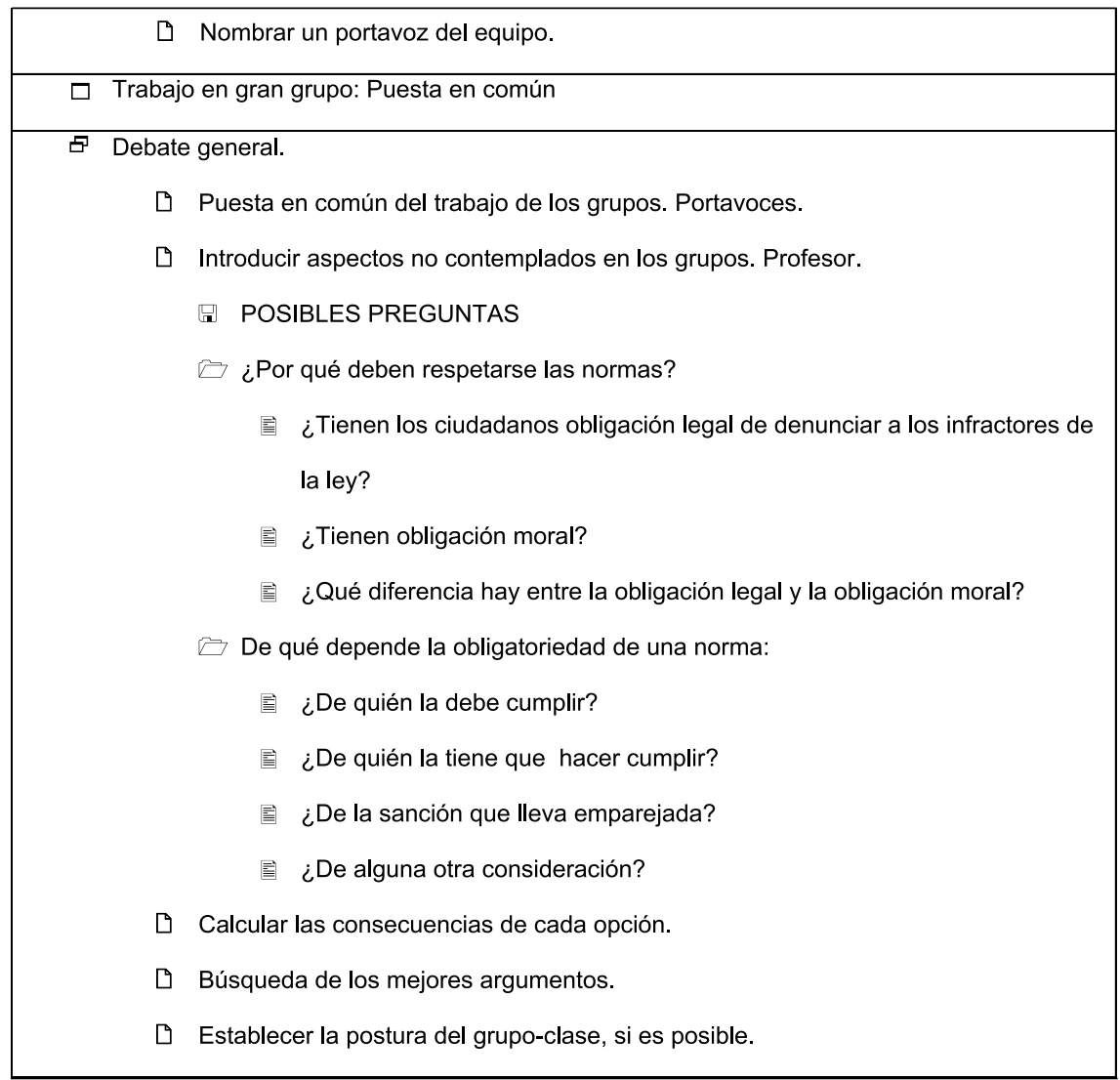

ACT.1 / IND

María iba por la calle y presencia un accidente en el que es atropellada una mujer. El conductor se da a la fuga, pero María ha retenido, además del modelo y color, la matrícula del vehículo.

Ahora se encuentra con el siguiente dilema:

Si se presenta como testigo del accidente y denuncia al conductor tendrá que ir a juicio, perder su tiempo, verse metida en líos, pasar nervios y, lo que todavía es peor, se enterarían sus padres del lugar donde se encontraba. Este último aspecto le supondría un disgusto en el ámbito familiar con consecuencias en sus salidas de casa y horarios, ya que sus padres le habían prohibido, expresamente, frecuentar la zona donde se ha producido el accidente.

Pero, por otra parte, si no se presenta como testigo y no lo denuncia deja de ayudar a la mujer pues no se hará justicia con ella. 
Revista de

investigaciones UNAD

Volumen 12. Número 1. Junio 2013

-Analiza cada una de las opciones que se le plantean a María:

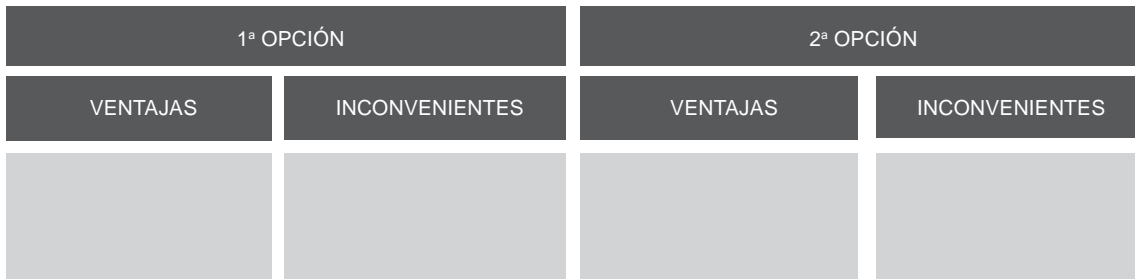

Analizada la situación, ahora, tienes que tomar una decisión.

¿Qué debe hacer María?

¿Por qué? Escribir las razones que justifican la alternativa elegida.

ACT.1 / EQUIPO

- Expresad la propia opinión y escuchad las posiciones de los demás.

¿Qué debe hacer María?

Determinad la posición del equipo y las razones que la justifican.

¿Qué debe hacer María?

¿Por qué? Escribir las razones que justifican la alternativa elegida. 


\section{Roberto Sanz Ponce y José M. Mula Benavent}

Un programa de educación en valores.

La formación moral en alumnos de educación secundaria obligatoria. Artículo de reflexión.

\section{-Comparad las siguientes normas:}

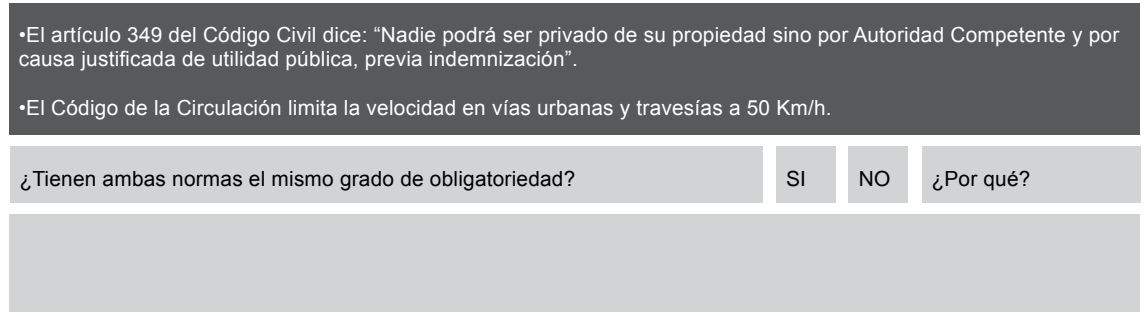

- Nombra un portavoz del equipo

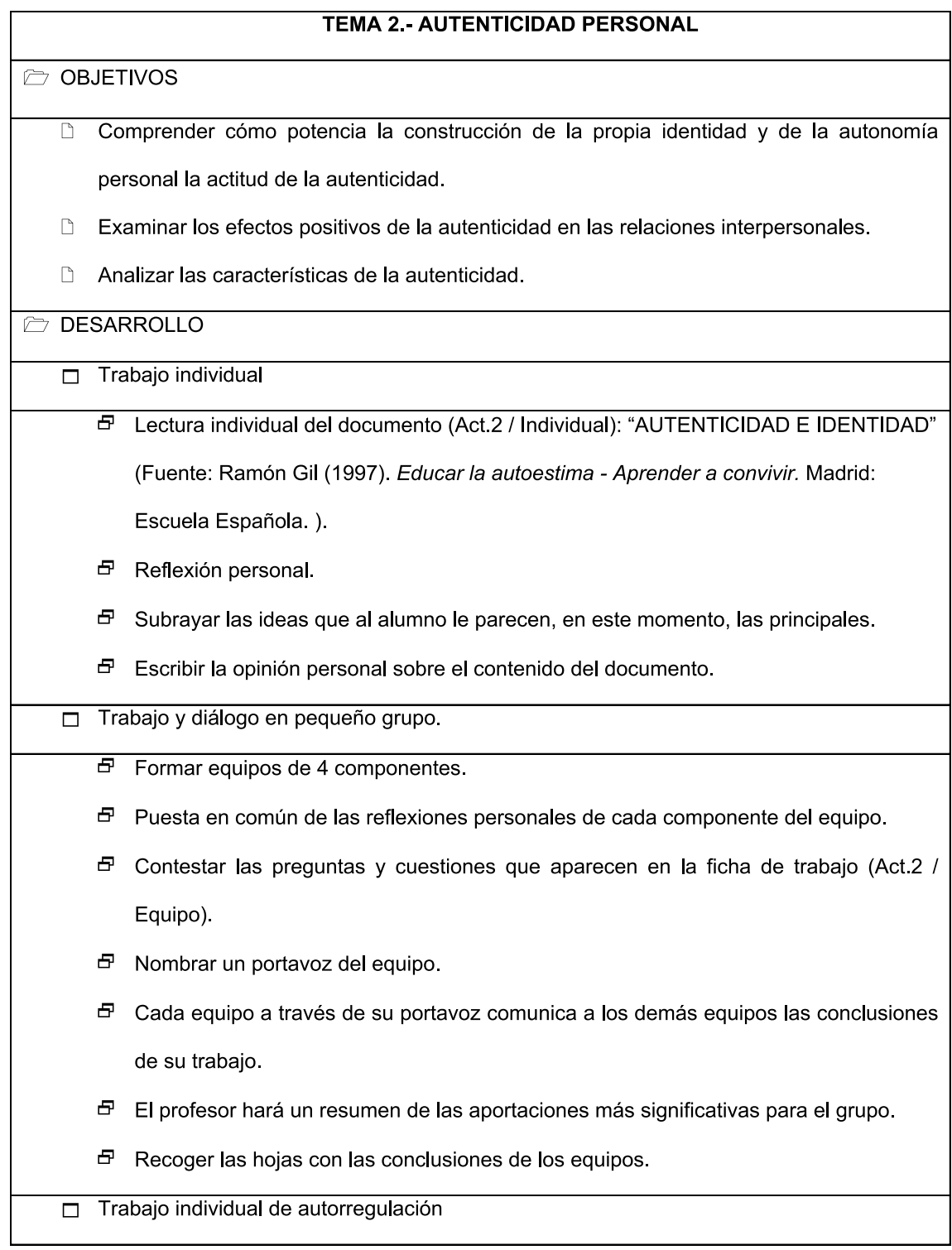




\section{Revista de}

\section{investigaciones UNAD}

Volumen 12. Número 1. Junio 2013

a Se entrega a los alumnos la ficha de trabajo Act.2 / Auto.

a Contestar de manera personal a las cuestiones que aparecen en ella.

a Rellenar, por duplicado, el documento de compromiso personal, aquellos que libremente quieran asumirlo.

다 profesor recogerá las copias del compromiso de aquellos alumnos que hayan suscrito el documento de compromiso.

ACT.2 / EQUIPO

TRABAJO Y DIÁLOGO EN PEQUEÑO GRUPO

Explicad lo que entendéis por:

- Autenticidad

- Aceptación de sí mismo.

- Coherencia.

- Ser uno mismo.

¿Pensáis que la autenticidad inspira confianza? ¿Por qué?

Aportad hechos y experiencias de qué es vivir

auténticamente

artificialmente 


\section{Roberto Sanz Ponce y José M. Mula Benavent}

Un programa de educación en valores.

La formación moral en alumnos de educación secundaria obligatoria. Artículo de reflexión.

ACT.2 / AUTO

AUTORREGULACIÓN DE CONDUCTA

¿Puedes ser suficientemente fuerte como para distinguirte de los otros (no ser masa) y no tener necesidad de depender de ellos (no sometimiento)?

Completa las frases siguientes:

- Soy auténtico cuando ...

- No soy auténtico si ...

- Soy coherente cuando

- No soy coherente si ...

- Para ser auténtico estoy

dispuesto a ...

- Para ser coherente me

comprometo a ...

Yo,

decido libremente llevar a la práctica durante el compromiso manifestado

Fecha y firma

TEMA 3.- MADUREZ HUMANA

\section{OBJETIVOS}

ㅁ Ofrecer cauces concretos para el desarrollo personal.

ㅁ Conocer algunas dimensiones o criterios para evaluar nuestro grado de maduración humana.

a Comprender que el desarrollo de la autoestima implica un proceso de madurez personal no exento de dificultades.

\section{DESARROLLO}

Trabajo individual

F Lectura individual del documento (Act.12 / Individual): "MADURACIÓN HUMANA" (Fuente:

Ginel, 1991: 19-28; \& Ramón Gil, 1997: 70-72)

ㅁ Reflexión personal.

Subrayar en el texto al menos tres ideas que el alumno considere, en este momento:

$\square \quad$ Novedosas para él. 


\begin{tabular}{|c|c|c|}
\hline 古 & $\mathrm{Escl}$ & $\begin{array}{l}\square \quad \text { No está nada de acuerdo con ellas. } \\
\square \quad \text { Está totalmente de acuerdo. } \\
\text { ribir la opinión personal sobre el contenido del documento. Reflexión personal. }\end{array}$ \\
\hline$\square$ & Tra & bajo y diálogo en pequeño grupo. \\
\hline & 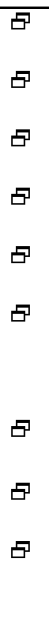 & $\begin{array}{l}\text { Formar binas. } \\
\text { Aportar el trabajo personal en cada uno de los apartados, justificándolo. } \\
\text { Dialogar sobre las aportaciones: coincidencias, diferencias,... } \\
\text { Formar equipos de } 4 \text { componentes ( } 2 \text { binas). } \\
\text { Puesta en común de las reflexiones llevadas a cabo en la fase de binas. } \\
\text { Consensuar un listado único con el que estén de acuerdo todos los componentes del } \\
\text { equipo. } \\
\text { Hacer un documento con el listado definitivo. } \\
\text { Colocarlo en la corchera del aula para darlo a conocer al grupo. } \\
\text { El profesor hará un análisis-resumen de las aportaciones más significativas para el } \\
\text { grupo. }\end{array}$ \\
\hline$\square$ & Tra & bajo individual de autorregulación \\
\hline & ロ & $\begin{array}{l}\text { Se entrega a los alumnos la Guía de Auto-observación que corresponde a la Act.12 l } \\
\text { Auto. } \\
\text { Contestar de manera personal a las cuestiones que aparecen en el cuestionario } \\
\text { "ACTITUD DE MADUREZ Y AUTORREALIZACIÓN". } \\
\text { En grupos de cuatro (diferentes a los formados en la primera fase) comentar las } \\
\text { respectivas respuestas, destacando todo lo positivo que hayan encontrado, en sus } \\
\text { respuestas y en las de los otros miembros del equipo. } \\
\text { El profesor invitará, para cerrar la sesión, a quienes deseen comunicar al grupo su } \\
\text { reflexión final o su sentimiento al finalizar. }\end{array}$ \\
\hline
\end{tabular}

ACT.1 / EQUIPO
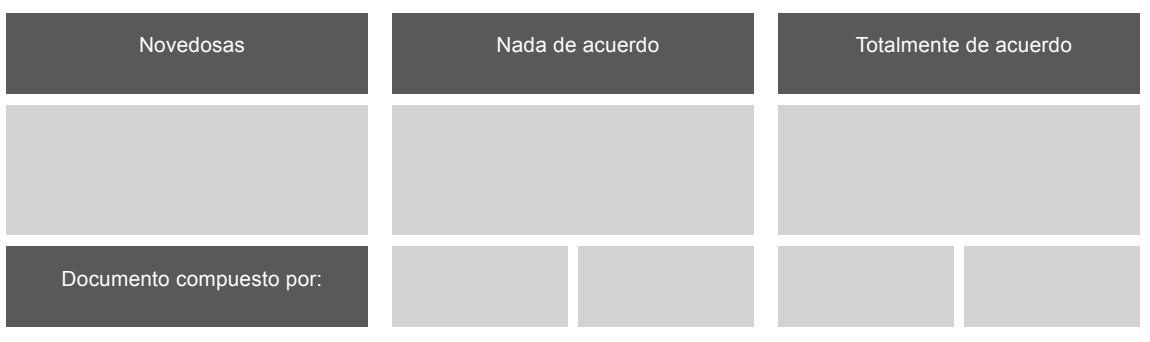


\section{Roberto Sanz Ponce y José M. Mula Benavent}

Un programa de educación en valores.

La formación moral en alumnos de educación secundaria obligatoria. Artículo de reflexión.

ACT.2 / AUTO

\section{GUÍA DE AUTOOBSERVACIÓN: ACTITUD DE MADUREZ Y AUTORREALIZACIÓN}

Cumplimenta el siguiente cuestionario.

Rodea con un círculo la categoría que refleje mejor tu situación personal:

$1=$ Raramente 2 = Algunas veces $3=$ Frecuentemente $4=$ Siempre

\begin{tabular}{|c|c|}
\hline 1. ¿Actúo con lealtad a la palabra dada? & $\begin{array}{llll}1 & 2 & 3 & 4\end{array}$ \\
\hline $\begin{array}{l}\text { 2. ¿Busco ser auténtico, rechazando cuanto huele a hipocresía, falsedad y } \\
\text { ambigüedad en mi manera de hablar y de actuar? }\end{array}$ & 12234 \\
\hline 3. ¿Rindo lo que debiera según mi capacidad? & 12334 \\
\hline $\begin{array}{l}\text { 4. ¿Actúo por convicciones propias, serenas, reflejas y no por caprichos y } \\
\text { fantasias? }\end{array}$ & 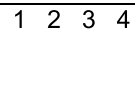 \\
\hline 5. ¿Procuro no ser precipitado, veleta e improvisador habitual? & 12234 \\
\hline 6. ¿Controlo y encauzo mi temperamento, sin excusarme con que «soy así»? & 12234 \\
\hline $\begin{array}{l}\text { 7. ¿Me esfuerzo en no dejarme llevar por los estados de excesiva euforia ni por } \\
\text { los de abatimiento? }\end{array}$ & $1223 \quad 4$ \\
\hline 8. ¿Normalmente no me vence el desaliento? & 12234 \\
\hline 9. ¿Busco no instalarme en un ritmo de vida fácil y rutinario? & 122334 \\
\hline 10. ¿Jamás utilizo a las personas "cosificándolas"? & 12234 \\
\hline $\begin{array}{l}\text { 11. ¿No margino a las personas menos inteligentes, menos simpáticas o más } \\
\text { sencillas? }\end{array}$ & 12234 \\
\hline 12. ¿Trabajo tanto como los que están sujetos a un horario laboral? & 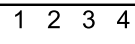 \\
\hline 13. ¿Suelo ser constante y dejar siempre acabadas mis tareas? & 12234 \\
\hline 14. ¿No soy vanidoso, fatuo ni pedante? & 12234 \\
\hline $\begin{array}{l}\text { 15. ¿Sé dialogar respetando las opiniones de los otros, sin pretender tener la } \\
\text { exclusiva de la verdad y de la razón? }\end{array}$ & 12234 \\
\hline 16. ¿No me escondo en la defensa de la verdad y la justicia? & 12234 \\
\hline 17. ¿Tengo una mente ágil, capaz de cambio y de evolución? & 12234 \\
\hline 18. ¿Soy solidario con el sufrimiento y necesidad ajenos? & 12234 \\
\hline $\begin{array}{l}\text { 19. ¿Procuro no ser superficial, ni dejarme llevar por lo que todos hacen, dicen o } \\
\text { piensan? }\end{array}$ & 12234 \\
\hline 20. ¿No suelo caer en el pesimismo, el desaliento, la tristeza y la desilusión? & 12234 \\
\hline 21. ¿No me alegro con los fracasos y humillaciones de los otros? & 12234 \\
\hline
\end{tabular}




\begin{tabular}{|c|c|}
\hline $\begin{array}{l}\text { 22. ¿Procuro no ser individualista y desarrollar actitudes de cooperación, } \\
\text { participación y colaboración? }\end{array}$ & 123 \\
\hline $\begin{array}{l}\text { 23. ¿No eludo la propia responsabilidad, ni me excuso echando la culpa a los } \\
\text { demás? }\end{array}$ & 123 \\
\hline $\begin{array}{l}\text { 24. ¿Tengo espíritu de superación y no he puesto un stop en el camino de mi } \\
\text { propia autorrealización? }\end{array}$ & 123 \\
\hline
\end{tabular}

En el nivel de los profesores que intervienen en el programa los temas por desarrollar en las sesiones y los objetivos de los mismos serían:

Los educadores referentes significativos de valores cívicos.

\section{Objetivos}

*Tomar conciencia del valor de ciertas conductas, por parte de los profesores, de cara a crear un clima de participación en el aula, para aprender a vivir juntos.

*Determinar las actitudes y conductas que facilitan el aprendizaje de la ciudadanía.

* Analizar ciertas conductas que favorecen y promueven la integración y la participación democrática, así como la coherencia en el actuar.

\section{Educación en la ciudadanía y prevención de la violencia.}

\section{Objetivos}

* Promover una convivencia pacífica, orientada a evitar actitudes y conductas de agresividad y de violencia, de racismo y de xenofobia, fomentando actitudes y conductas alternativas.

*Estimular las tendencias altruistas naturales hacia la bondad, la compasión, la generosidad y la empatía como prevención de la agresividad y de la violencia.

Las sesiones de trabajo con los padres de los alumnos se desarrollan con la metodología de la Escuela de Padres, entendida ésta como una escuela activa, por lo tanto, siguiendo los principios de la misma. Los temas por desarrollar en las sesiones y los objetivos de los mismos serían: 


\section{La familia como escuela de ciudadanía}

\section{Objetivo}

*Tomar conciencia de que el clima de afecto, de aceptación y de comprensión que debe presidir las relaciones familiares constituye una experiencia fundamental en el aprendizaje de la ciudadanía.

\section{Diálogo y convivencia en el ámbito familiar}

\section{Objetivos}

*Identificar las actitudes que favorecen el diálogo, la participación y la convivencia en la familia.

*Favorecer un clima positivo de convivencia y comunicación en la familia.

\section{4.- La evaluación de los valores en la escuela}

Una de las tareas más complejas a las que se enfrentan los docentes es la evaluación de los aprendizajes de los alumnos. Esta tarea, es aún más compleja cuando nos enfrentamos con la evaluación de los contenidos actitudinales o como se ha venido llamando a lo largo de este artículo, de los valores.

Toda evaluación es una actividad de carácter subjetivo, los contenidos que se evalúan, la forma en la que se hace, el momento de la prueba, todo ello, son decisiones que se toman de manera personal, en función de unas ideas o principios, por tanto, de manera subjetiva. Pero, históricamente, se ha tenido la falsa percepción de que la evaluación de los contenidos conceptuales conocimientos- tenía un carácter objetivo-científico -incluso se crearon las denominadas pruebas objetivas en referencia a los exámenes tipo test; - en contraposición con la evaluación de los contenidos actitudinales que estaba marcada por aspectos secundarios, tales como: que te cae bien el alumno, que se porte bien en clase, que no moleste mucho, que haga las tareas, que se relacionaban con aspectos más cercanos a lo afectivo-emocional.

Para romper esta falsa percepción los docentes deben enfrentarse a dos grandes inconvenientes a la hora de afrontar la evaluación actitudinal. Por un lado, acabar con aquellos problemas derivados del carácter ético-filosófico de los contenidos presentados. Evaluar si una conducta es buena o mala, si está bien o mal, no siempre es una tarea sencilla y admitida de buen gusto por los propios docentes -no se consideran moralizadores. Por otro lado, los problemas derivados de las cuestiones técnico-metodológicas, entendidas como 
la falta de formación y de conocimiento en y de las técnicas e instrumentos más adecuados para este tipo de evaluación.

Estas carencias deben ser superadas buscando un cierto equilibrio entre la importancia que le se le dé a estos contenidos durante el proceso de aprendizaje-enseñanza (que debe ser mucha) y el peso real que le otorguemos en la evaluación y en la calificación de nuestros estudiantes. Por otro lado, se debe dar a conocer, con claridad, los criterios, momentos, instrumentos y procedimientos que van a ser utilizados para trabajar y evaluar los valores. Por último, en referencia a las técnicas e instrumentos más adecuados para evaluar los valores, los docentes deben formarse en técnicas tales como: la observación directa y sistemática; las rúbricas; las hojas de registro y el registro anecdótico, entre otros. Además, deben entender la evaluación como una parte más del proceso, no separada ni ajena a este.

\section{Conclusiones}

La educación en valores creemos que es una necesidad imperiosa con alumnos de Educación Secundaria Obligatoria en una sociedad como la actual. El descrédito de ciertos principios ético-morales; la influencia de los medios de comunicación, preocupados no precisamente por la educación de los más jóvenes; la cada día más creciente sociedad consumista; la radicalización, por causa de la crisis económica, de ciertas posturas políticas de carácter extremista; la ruptura cada vez mayor entre el primer mundo y el segundo, tercero y cuarto; hacen de este tipo de educación una "urgencia educativa."

El hecho de centrarnos en alumnos de Educación Secundaria Obligatoria, con edades comprendidas entre los 12 y los 16 años, tampoco es una cuestión baladí. Los adolescentes se enfrentan a un gran número de estímulos y de decisiones que pueden marcar de forma dramática su vida - consumo de drogas, descuido por los hábitos de vida saludable Por ello, instaurar un Programa de educación en valores en estas edades, pensamos que es muy oportuno.

En esa misma línea, llevarlo a cabo dentro del ámbito escolar, introduciéndolo en el propio curriculum académico de los alumnos, es una decisión que responde a dos cuestiones fundamentales: por una parte, es una cuestión de ley, es decir, de cumplimiento de la Ley Orgánica de Educación (LOE) que recoge entre su articulado la obligación y la necesidad de educar en valores; y, por otro lado, es una cuestión de calidad. Poner la educación en valores en manos de los docentes, verdaderos especialistas en la creación de situaciones de aprendizaje, dota a esta educación de ciertos parámetros de calidad.

Por último, la elección, en este primer bloque, de los valores: 1) El respeto a las normas; 2) Autenticidad personal; y 3) Madurez humana, tampoco ha sido 


\section{Roberto Sanz Ponce y José M. Mula Benavent}

Un programa de educación en valores.

La formación moral en alumnos de educación secundaria obligatoria. Artículo de reflexión.

una cuestión de azar. La decisión de establecer en $1^{\circ}$ de Educación Secundaria Obligatoria este Programa responde a la necesidad de instaurar entre nuestros alumnos la capacidad de conocer, consensuar y cumplir una serie de normas que ayudan, facilitan y benefician la convivencia del grupo. Además, prepararlos para tomar sus propias decisiones, sin coacciones ni presiones, siendo ellos mismos y responsabilizándose de sus actos y decisiones, demostrando un cierto grado de madurez personal.

\section{Referencias bibliográficas}

Bartolomé, M. et al. (1979). Educación y valores. Sobre el sentido de la acción educativa en nuestro tiempo. Madrid: Narcea.

Carreras, LL. \& otros (1996). Cómo educar en valores. Materiales, textos, recursos y técnicas. Madrid: Narcea.

Cortina, A.; Escámez, J. \& Pérez-Delgado, E. (1996). Un mundo de valores. Valencia: Generalitat Valenciana.

Dewey, J. (1995). Democracia y educación. Una introducción a la Filosofía de la Educación. Madrid: Morata.

Escámez, J. \& Gil, R. (2001). La educación en la responsabilidad. Barcelona: Paidós.

Escámez, J. \& Ortega, P. (1986). La enseñanza de actitudes y valores. Valencia: Nau Llibres.

Escámez, J. \& otros (1998). Educar en la autonomía moral. Valencia: Generalitat Valenciana.

Gil, R. (1998). Valores Humanos y Desarrollo Personal. Madrid: Escuela Española.

Ginel, A. (Coord.). (1991). Formación Básica, 1. Madrid: CCS.

Llopis, J.A. \& Ballester, Ma R. (2001). Valores y actitudes en la educación. Teorías y estrategias educativas. Valencia: Tirant lo Blanch.

Marín, R. (1976). Valores, objetivos y actitudes en educación. Valladolid: Miñón.

Martín, X. \& Puig Rovira, J.M. (2007). Las siete competencias básicas para educar en valores. Barcelona: Graó. 
Mula, J. M. (2007). Educar en valores: una forma de hacer escuela. En Educa Nova. Colección de artículos técnicos de educación (1), 89-107.

Piaget, J. (1974). A donde va la educación. Barcelona: Teide.

Puig Rovira, J. M.; Martín, X. \& Trilla, J. (1998). Cròniques per a una educació democràtica. Experiències de formació en valors a secundaria. Vic: Eumo.

Reboul, O. (2009). Filosofía de la Educación. Barcelona: Davinci.

Sanz, R. (2011). Sentido y finalidad de la educación secundaria en la sociedad española del siglo XXI. En Sinéctica (36-37), 141-152.

Vallet, M. (2011). Cómo educar a nuestros adolescentes. Un esfuerzo que merece la pena. Madrid: Wolters Kluwer.

Vinuesa, M $M^{\text {a }}$. (2002). Construir los valores. Curriculum con aprendizaje cooperativo. Bilbao: Descleé. 


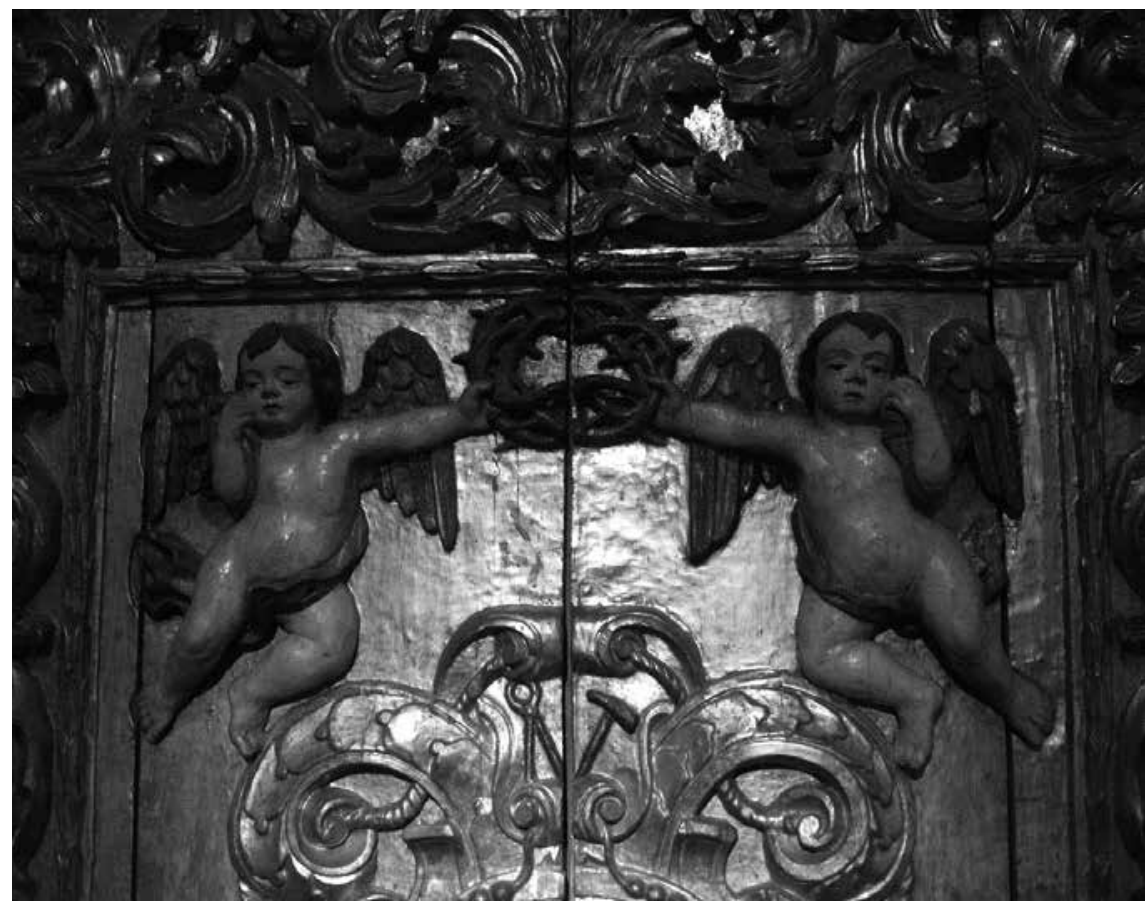

Fotografía: José Enrique Castillo 